\title{
HERRAMIENTAS DIGITALES \\ PARA EL DISEÑO BIOMIMÉTICO SUSTENTABLE
}

M.Eng. Ph.D. Valentina Villa

\begin{abstract}
Department of Structural, Geotechnical and Building Engineering (DISEG). Universidad Politécnica de Turín. Italia.

E-mail: vale.villa@gmail.com
\end{abstract}

A finales del siglo XIX nace la "epistemología la complejidad" en contraste con el pensamiento del siglo XVIII y al dualismo cartesiano, ya inadecuados a comprender y explicar las ciencias modernas, en particular la física, así como expuesto por el premio Nobel de física de 1969, Murray Gell-Mann. [1] En el mismo campo de investigación epistemológico se asiste también a una pérdida del concepto de "certeza absoluta" típica del determinismo y una toma de conciencia de la incertidumbre o indeterminismo inherente en toda la ciencia moderna a partir de la física moderna. En particular, el estudio del comportamiento y de la evolución de los sistemas complejos ha tenido consecuencias interesantes sobre la filosofía, sobre la economía y, en estos últimos decenios, también sobre la arquitectura. Una crisis de la explicación simple ha invertido las ciencias físicas y biológicas "consecuencia", observa Edgard Morin, "los que parecen ser los residuos no científicos de las ciencias humanas - la incertidumbre, el desorden, la contradicción, la pluralidad, la complicación, etc. - Forman hoy parte de la problemática de fondo del conocimiento científico". [2]. La comprensión de las estructuras orgánicas, sistémicas, se está convirtiendo en una de los desafíos para diversas disciplinas modernas, entre ellas la arquitectura. La mayor complejidad, de sistemas, procesos y formas, siempre ha sido dada por la naturaleza, principal fuente de inspiración de todos los estudios científicos, a partir de Leonardo y los más grandes maestros de la historia. "Cada cosa que puedes imaginar, la naturaleza la ha creado", sostenía Albert Einstein y esta línea de pensamiento fue abrazada por todas las ciencias artísticas incluidas la arquitectura y el diseño. La imitación de las formas y los comportamientos de la naturaleza ha sido enormemente facilitada, en estos últimos años, las posibilidades fechas las evoluciones tecnológicas y la teoría geométrica de los Fractales, lenguajes de programación y codificación, modelado e impresión 3D permiten en efecto, reproducir, de manera fiel estructuras, geometrías, líneas y labores de organismos naturales, caracterizados por una evolución milenaria de adaptación evolutivo típico de los hábitats naturales. Este es el campo en que se mueve la biomimética, una ciencia relativamente joven que expresa el vínculo entre biología y tecnología, donde la primera representa el modelo primordial a la que tender y la segunda el medio con que reproducir el funcionamiento en materiales y estructuras. [3] El estudio de los procesos naturales se convierte en fuente de inspiración para la mejora de las tecnologías constructivas, del confort de la vivienda y las actividades humanas, como sostiene en su libro "Biomimicry in Architecture", Michael Pawlyn. [4] Con la tecnología se están abriendo innumerables escenarios pero el abanico de posibilidades tecnológicas es tan amplio que no dice nada sobre la eficacia de sus usos, dado que depende de la lectura crítica y de las capacidades interpretativas de los usuarios.

\section{Las fases de vida de la construcción}

El ciclo de vida de un elemento natural se caracteriza por la falta de residuos. Durante milenios también el hombre ha hecho parte de este proceso, integrándose en modo perfecto con lo que le rodeaban, sin afectar o alterar sistemas perfeccionados por millones de años de existencia, donde todo nace, crece, muere y se transforma en algo diferente. La naturaleza realiza un continuo y completo reciclado para que todo lo que se rechaza por un proceso se transforma en un recurso para otros organismos. La naturaleza se define en efecto, un ciclo cerrado (Fig. 1a). 
Con el nacimiento de la industria química, el ciclo natural se abre, porque el hombre crea nuevos productos sintéticos y no biodegradables. La naturaleza resulta incapaz de reciclarlos en tiempos 'breves' y el hombre no desarrolla una tecnología suficientemente biosostenible para poder cerrar el círculo. Comienza pues, un proceso inexorable y que asume proporciones gigantescas de continua extracción de la tierra de muchas materias primas, de construcción a escala industrial de objetos que, al término de su vida útil, se eliminan sin un proceso de recuperación y reciclado completo (Fig. 1b)

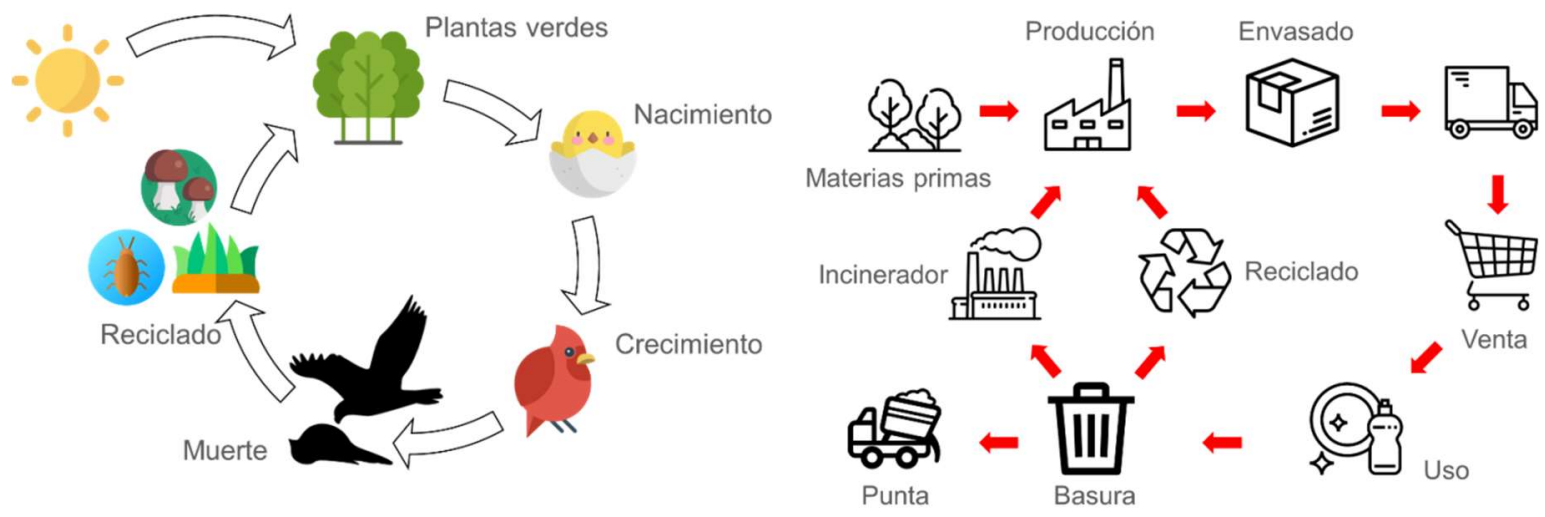

Fig. 1 - Ciclo de vida natural (a) y ciclo de vida de los productos (b)

En los años '90 del siglo pasado, se ha introducido un método que permite evaluar "el ciclo de vida" (Life Cycle Assessment) de un producto o de un servicio en base a las interacciones y los procesos que contribuyen para su realización, manipulación, utilización y eliminación. El Life Cycle Assessment considera por primera vez todo el ciclo de vida, incluyendo por lo tanto las fases de preproducción (extracción y transformación de materias primas), producción, distribución, uso (incluido su reutilización y mantenimiento), reciclaje y separación final. [5] El procedimiento LCA ha sido normalizado a nivel internacional de las normas ISO 14040 (gestión ambiental, evaluación del ciclo de vida, principios y marco de referencia) e ISO 14044 (evaluación del ciclo de vida, definición y líneas guía) para los productos industriales, y se extendió también al sector de las construcciones con la EN 15804 (Sustainability of Construction works). Esta norma define los instrumentos y los parámetros necesarios para medir el impacto medioambiental del proceso constructivo. La construcción es una fase del proceso de construcción todavía poco analizada principalmente a causa de su complejidad y número de unidades de los sujetos en juego. Es precisamente en esta fase que se usan medios muy contaminantes, se utilizan recursos valiosos, como agua y suelo, y se producen importantes cantidades de residuos.

El proceso de construcción se define como la secuencia organizativa de fases que llevan el rumbo de las necesidades de encargar, a su satisfacción, mediante el diseño, producción, construcción y gestión del propio bien (UNI 10838) (Fig. 2).

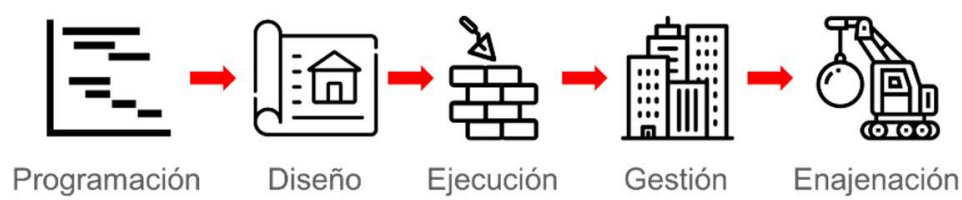

Fig. 2 - Proceso de construcción lineal tradicional

Estas fases son gestionadas por sujetos distintos (el comitente, el diseñador, las empresas ejecutores y el gestor o el usuario del bien) y se suceden siguiendo una lógica temporal secuencial, muy diferente de los ciclos naturales o de producto, que tienden a cerrar, aunque sólo en parte, un recorrido. En realidad, el ciclo de vida de un edificio es esencialmente similar al de un ser viviente: un comitente encuentra recursos para financiar una intervención, los diseñadores definen prestaciones y tecnologías constructivas, las empresas realizan las distintas partes de obra, el usuario disfrutará del edificio $\mathrm{y}$, al final, el mismo edificio podrá ser refuncionalizarlo o demolerlo. William McDonough y 
Michael Braungart [6] Proponen un ciclo que va 'de la cuna de la cuna', es decir, un recorrido continuo que desde finales de vida de un producto imagina el comienzo de un nuevo producto, evitando o al menos limitando la producción de residuos. Se trata de una contribución muy importante en el campo tecnológico y de diseño, sobre todo porque propone transformar un problema en una oportunidad. El final de su vida de un edificio, se convierte en el comienzo de otro; un paso más hacia la definición de un mundo de las construcciones y una arquitectura ecosistémica.

\section{Una nueva metodología en el sector AEC: Building Information Modeling}

Esta nueva visión cíclica del proceso de construcción se hizo posible a partir de la introducción de una nueva metodología, el Building Information Modeling. El BIM es innovación de proceso, método y herramienta de planeamiento y gestión que afecta a todos los actores del proceso de construcción. La definición que da Eastman es significativa y subraya el aspecto metodológico de la cuestión: "El BIM no es una cosa o un tipo de software, sino una actividad humana que conlleva, en última instancia, amplias modificaciones de los procesos en el sector de la construcción". [7]

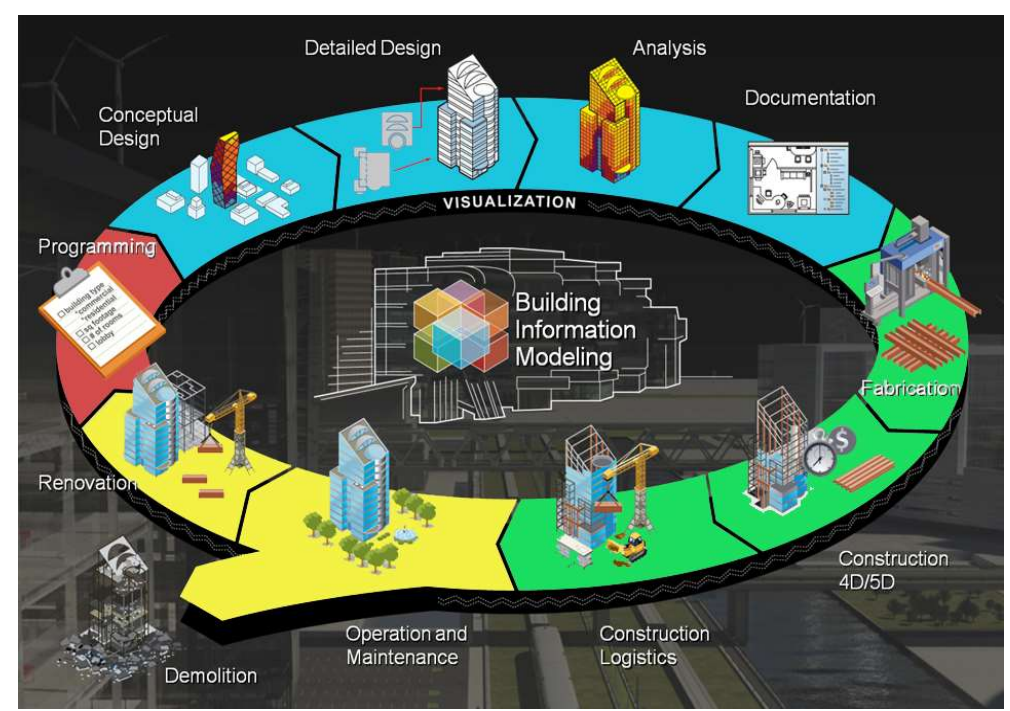

Fig. 3 - Ciclo de vida de un edificio con la metodologia BIM. Fuente: Autodesk.

El acrónimo BIM es de origen inglés y asume diversos significados principalmente en relación con los objetivos, la complejidad del sistema en el que se inserta, pero lo más significativo y más amplio es el de Management: "La organización y control del proceso empresarial utilizando las informaciones del prototipo digital para efectuar el intercambio de información sobre todo el ciclo de vida de un bien. Los beneficios incluyen: comunicación centralizada y visual, exploración inicial de alternativas, sostenibilidad, eficiencia en el diseño, integración de disciplinas, control del obrador, documentación conforme a obra, etc.; desarrollando eficazmente el proceso del ciclo de vida de un bien y el modelo desde la concepción al desguace" (BuildingSMART).

En este caso el BIM puede ser utilizado como un instrumento de gestión del ciclo de vida (Fig. 3) del edificio, para facilitar flujos de trabajo y procedimientos para que los diversos actores provean con cargo información comprobable en cualquier fase del proceso de construcción. Dada la excesiva fragmentación del proceso de construcción y la limitada coordinación entre los sujetos implicados, uno de los grandes ventajas en el uso de la metodología BIM es el de elaborar un modelo virtual capaz de contener todas las informaciones necesarias al proyecto. [8]

EI BIM no sólo representa la introducción de una nueva ICT (Information and Communication Technology), sino que también implica la definición de nuevas metodologías de trabajo soportadas por una mejor coordinación e integración entre los actores del proceso de construcción y una puesta a punto de soluciones innovadoras relativas a la organización y a los hábitos de trabajo por tiempo consolidadas. En consecuencia, el BIM no significa la utilización de un nuevo software sino que 
implica un cambio radical en los procesos de trabajo y por tanto un enfoque al diseño, construcción y gestión de la obra distinta de la tradicional. Las dos características principales de este sistema son la modelización paramétrica de los objetos y la creación de una única base de datos.

Por primera vez tenemos a disposición una base de datos relacional de la información del edificio, vinculadas a los objetos de modelado que representan el alter-ego digital del edificio en todas sus partes. Es posible gestionar las distintas partes de la complejidad de las relaciones que inevitablemente se crean en la fase constructiva y gestionar, paralelamente, el ciclo de vida del edificio. La creación de un modelo virtual que reproduce, en todas sus partes, los sistemas que componen un edificio (arquitectónico, estructural e instalaciones) permite aplicar una revolución metodológica en el ámbito del diseño. Actualmente el proceso de diseño prevé la definición de un primer concepto, y la verificación de viabilidad (económica, espacial, distributiva, energética, etc.) del mismo, aplicando el control sobre el proyecto. La informática en efecto, como veremos posteriormente, es un instrumento complejo capaz de reproducir sistemas complejos, por este motivo es el mismo modelo digital que ofrece más soluciones de diseño en relación con los insumos de proyecto que se suministran el profesional, traduciendo los requisitos y las especificaciones de proyecto en elementos informativos útiles para la definición del proyecto. Variando cualquiera de los parámetros iniciales se pueden generar recorridos alternativos, más o menos diversificados. La consecuencia es que el recorrido de diseño de un edificio remite siempre "a una amplia gama, diríamos a un haz de caminos paralelos: lo que no ha sido, sino que también podía ser. Diríamos que el arquitecto contemporáneo debería tomar conciencia de que el objeto de su investigación no es sólo el real, pero también el 'posible', es decir, una familia de mundos posibles en la que de vez en cuando aparecen actualizados de los particulares subsistemas, según las opciones y los objetivos a corto y largo plazo". [9]

\section{Diseño paramétrico y computacional}

La informática, vocablo que nace de la contracción de "información automática", es una disciplina que estudia los fundamentos teóricos de la información y los medios que hacen posible la implementación y su transmisión. Representa el vínculo entre dos ciencias autónomas: la electrónica y la matemática o más exactamente la algorítmica. La informática, en efecto, se ocupa del estudio sistemático de los procesos algorítmicos que describen y transforman la información a través de máquinas representación y procesamiento de números. El centro de la revolución informática, por tanto, no está constituido tanto por la información, desde su inmenso número o la perenne mutabilidad, lo que la capacidad de los átomos informativos de ser interconectados, interrelacionados para formar precisamente una red y un sistema.

La disciplina que se ocupa de este se define con varios términos: Computational Design, Parametric Design, Numerique. Cada uno de estos términos describe características fundamentales de la disciplina. Diseño propiamente significa diseñar. Computational se refiere a los cálculos efectuados por ordenador: los cálculos en la base de este método son así articulados que el ordenador no es sólo un instrumento de ayuda, capaz de mejorar, agilizar y simplificar, sino que también es el único capaz de recorrer este método de diseño. Parametric aclara que esta modalidad de diseño ocurre con los parámetros: se trata de parámetros numéricos, que es posible variar para obtener una distinta configuración del proyecto. Los números en cuestión pueden corresponder a datos dimensionales, estructurales, térmicos y así sucesivamente. Esto es posible porque en este método el trabajo del diseñador se expresa en la elaboración de informes algebraicos que transcurren entre las partes y no solo en la composición de las formas geométricas. Se trata de un enfoque innovador, complejo, aún no accesible al gran número de arquitectos, y por tanto poco comprendido y a menudo usado en forma abusiva o incompleta, pero es evidente que "... los problemas cruciales, problemas como energía, medio ambiente, cambio climático, pobreza, no pueden ser comprendidos separadamente, son problemas sistémicos, interconectados e interdependientes". [10]. El diseño requiere por tanto, cada vez más a menudo, la referencia a parámetros de las prestaciones y el uso de la modelización paramétrica para la evaluación de toda la complejidad para la determinación de las formas.

La definición de un proyecto generativo se realizará por medio de software paramétricos (computational design): como sucede en la materia biológica, en que la inmanencia de los caracteres 
se elaborada con un conjunto de reglas (ADN) para la determinación del organismo, así, con los programas paramétricos es posible trabajar con las relaciones entre los elementos, en forma de establecer un conjunto de pasos algebraicos que definen la matriz que se podrá sacar un número variable de proyectos actualizados. Pues con los software de modelización para la arquitectura de los datos en que se trabaja son principalmente numéricos, los parámetros pueden corresponder a las dimensiones, cantidad y a todas las relaciones entre ellos.

El hecho de que el proyecto generativo se base en datos numéricos, sin embargo, no debe confundir y hacer identificar este instrumento como un generador cantidad de soluciones indistintas: el uso de un modelo de diseño demanda - prestación se basa en la superación de un enfoque cuantitativo en beneficio de un enfoque cualitativo. La posibilidad de poder interactuar con parámetros específicos hace personalizable el proyecto y por tanto la idea de calidad, en este enfoque, se define precisamente por el valor de la personalización. Es de este modo que se establece un control a priori del proyecto: es la solución, en términos de las prestaciones, que guía la forma del proyecto. El proyecto generativo es virtual, es decir, es una idea general que contiene en sí la multiplicidad.

\section{Instrumentos digitales para el diseño parametrico y la geometría fractal}

Aproximadamente un siglo antes del nacimiento de la geometría fractal el arquitecto Antoni Gaudì diseña formas ligadas de manera indisoluble al estudio y las formas de la naturaleza. "El gran libro, siempre abierto y que es preciso esforzarse de leer, es el de la naturaleza; los otros libros proceden de este y contienen, además, interpretaciones y malentendidos de los hombres. Hay dos revelaciones: una, la de los principios de la moral y la religión; la otra, que guía a través de los hechos, es la del gran libro de la naturaleza. Los aviones presentan una ordenación similar al de los insectos con alas planas y no rígidas que, desde hace siglos ya vuelan perfectamente. La construcción se trata de protegernos del sol y la lluvia, como el árbol que recoge el sol y la lluvia. La imitación [de la naturaleza] llega hasta las membrature arquitectónicas, desde el momento en que los árboles sirven de columnas; en un segundo momento vemos los capiteles adornados de hojas". [11] A partir de esta sugestión podemos identificar dos tendencias, desde siempre presentes en el ánimo de artistas y arquitectos respecto de la naturaleza: la simulación y la imitación. Por 'imitación' de la naturaleza se pretende asumir un comportamiento no original basado en un modelo anterior: la mimesis, en efecto, es un concepto de fundación de la creación artística y la naturaleza representa uno de los sujetos más "copiados" y que se encuentre más inspiración. Con 'simulación' de la naturaleza se entiende, en cambio, reproducir en forma artificial un fenómeno real que parezca verdad.

Las formas de la naturaleza son muy complejas, y articuladas y no ha sido siempre fácil estudiar, aprender a conocer, comprender y reproducir las formas y los procesos naturales. Aunque el vínculo entre la naturaleza y las matemáticas se intuía desde hacía siglos, Galileo Galilei, considerado universalmente como el padre del método científico, resumía su pensamiento de la siguiente manera: "El libro de la naturaleza está escrito en lenguaje matemático y sus caracteres son triángulos, círculos $y$ otras figuras geométricas, sin las cuales es imposible comprender humanamente su palabra; sin ellas es un vano deambular por un oscuro laberinto". La geometría tradicional no es capaz de manejar las reglas y la complejidad típicas de la naturaleza. De hecho, el matemático polaco Benoit Mandelbrot escribe: "La geometría euclidiana es incapaz de describir la naturaleza en su complejidad, ya que simplemente describe todo lo que es regular. Todos los objetos que tienen una forma perfectamente esférica, mientras observamos la naturaleza vemos que las montañas no son conos, las nubes no son esferas, las costillas no son círculos, sino objetos geométricamente muy complejos." (de Les objects fractals 1975). A diferencia de la geometría euclidiana, que ha intentado una interpretación abstracta de la realidad, generando orden y armonía, pero también simplificación y reducción, la geometría fractal es capaz de interpretar con mayor fidelidad el aparente desorden natural, sin descuidar la riqueza y la variedad. A principios del siglo XX, gracias a los estudios del matemático francés Gaston Julia, se menciona por primera vez la geometría fractal.

La palabra fractal fue acuñada por Beniot Mandelbrot en 1970, quien una década más tarde publicó "The fractal geometry of nature" (La geometría fractal de la naturaleza) (1982) donde teorizó las 
propiedades de los fractales como "código genético" de todos los sistemas naturales. Según su punto de vista, por lo tanto, la naturaleza es fractal hasta el punto de que hoy en día la geometría fractal se define como "la geometría de la naturaleza". Es con el advenimiento de las computadoras y de los sistemas electrónicos de cálculo que se hace posible representar y visualizar curvas y fractales, Paolo Portoghesi, en su "Naturaleza y Arquitectura" escribe: "Después de haber sido educado por el hombre para pretender ser un buen diseñador, el ordenador finalmente ha tenido una tarea a su altura: representar lo que la mente humana había sido capaz de concebir, pero que sus manos no habian sido capaces de traducir en formas para la excesiva complejidad de las operaciones necesarias". [12] Actualmente existe un gran número de herramientas digitales que permiten modelar geometrías complejas a partir de la entrada y manipulación de parámetros. Una de las herramientas más conocidas utilizadas en el modelado BIM es Dynamo $\AA$, un software de programación paramétrica visual que crea su propia geometría a partir de cadenas preestablecidas que procesan datos de entrada y salida. Dynamo ${ }^{\circledR}$, a diferencia de otros programas similares, lee y escribe en bases de datos externas y por lo tanto es perfecto para interactuar con otro software basado en BIM.

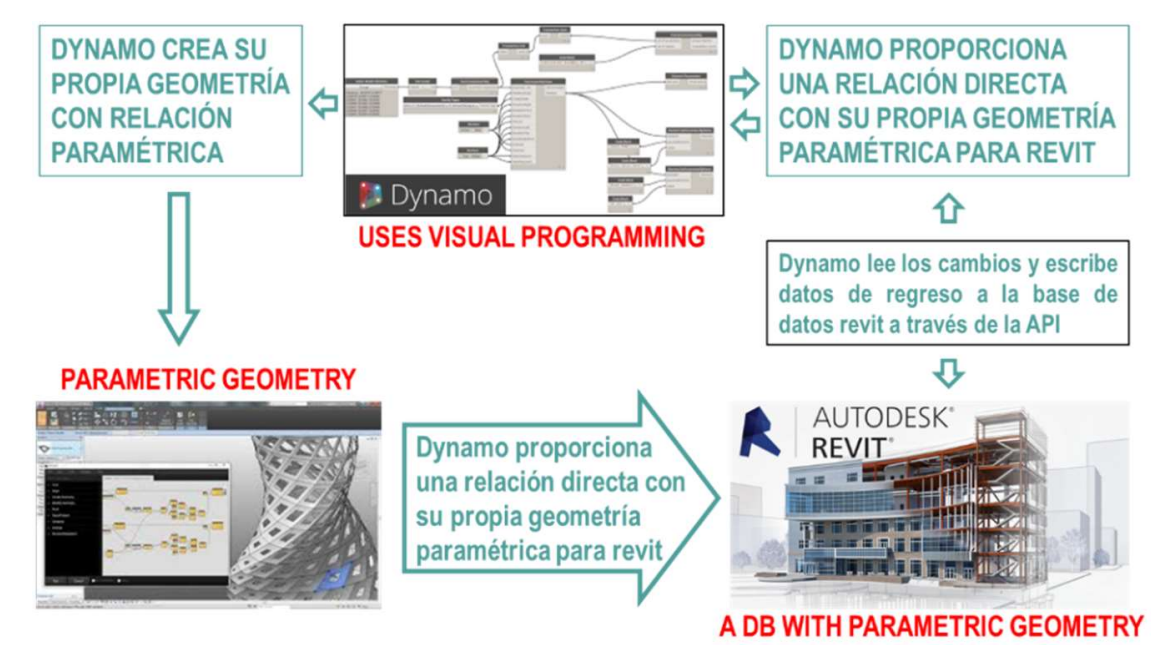

Fig. 4. Relación entre la Programación Visual y la Geometría Paramétrica (Marcello Sgambelluri) [13]

Es un programa independiente, pero se puede utilizar como un "complemento" para, por ejemplo, Autodesk Revit $\AA$. Dynamo actúa directamente sobre la API Revit $₫$, leyendo y escribiendo datos en la base de datos relacional BIM (Fig. 5). Los datos pueden ser casi cualquier cosa: valores de parámetros, geometría de familia y posicionamiento, etc. Básicamente, Dynamo® actúa sobre la base de datos relacional Revit $囚$ de maneras que no pueden ser manejadas a través de la interfaz de usuario. Dynamo ${ }^{\circledR}$ también proporciona una relación directa entre su geometría programada matemáticamente usando algoritmos y el modelo ya construido en Revit $₫$ creando un sistema de dos vías que es fácilmente actualizable, tanto en una dirección como en la otra. En la siguiente parte verás un posible uso de Dynamo® para la creación de geometrías fractales, que reproducen y copian formas naturales. En la naturaleza nada es perfectamente redondo, cuadrado o triangular, sino al contrario irregular y extremadamente complejo. (Fig. 5) 


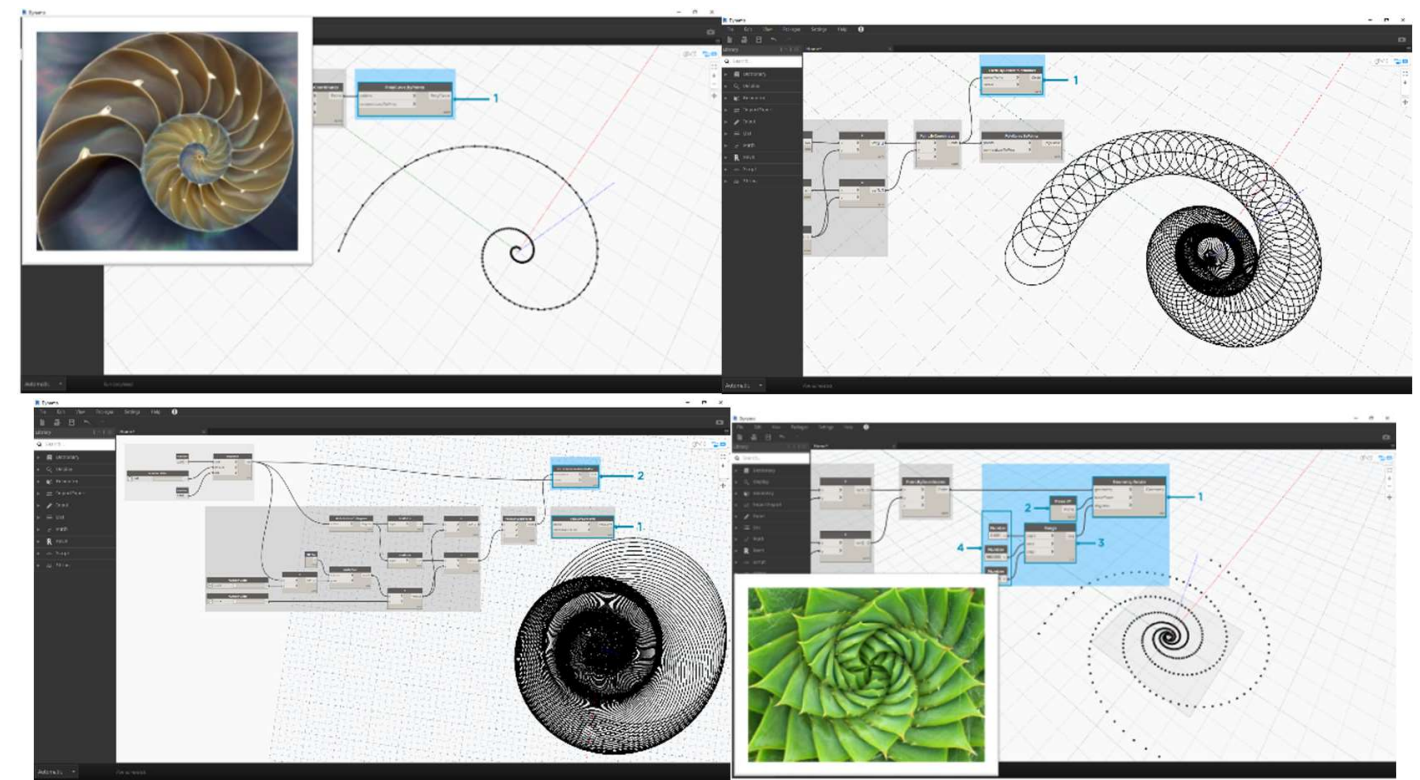

Fig. 5. Representación de geometría fractal con programación paramétrica (Fuente: WEB)

Árboles, inflorescencias, redes neuronales, líneas dentadas de costa, consideradas durante mucho tiempo formas anómalas e irregulares, y por lo tanto no describibles, son en realidad estructuras fractales donde las partes individuales tienen un alto grado de semejanza con el todo. Las formas fractales, de hecho, se generan a través de la reiteración de ecuaciones no lineales o, simplificando, repiten en una escala cada vez más pequeña su propia forma. Esta nueva geometría, por lo tanto, parece describir las formas y configuraciones de la naturaleza no sólo más fielmente, sino también estéticamente más válidas que la geometría euclidiana tradicional. Hay dos propiedades fundamentales de esta disciplina: la dimensión fractal y el interescalar autodidacta que representan ideas muy interesantes para la experimentación de métodos innovadores de diseño a diferentes escalas. Gracias a los ordenadores y a los potentes programas informáticos, se han podido identificar algunos factores y leyes importantes que conforman la Naturaleza y que actúan a la vez en diferentes niveles fundamentales e insustituibles para el éxito y la supervivencia del organismo, y sobre todo es posible reproducirlos artificialmente. El acercamiento a la Naturaleza, permanece como fuente de inspiración formal y emocional, pero sobre todo se convierte en un modelo a imitar en términos de equilibrio, ventaja, evolución y progreso: "La cáscara genera una forma mientras se genera a sí misma." [14].

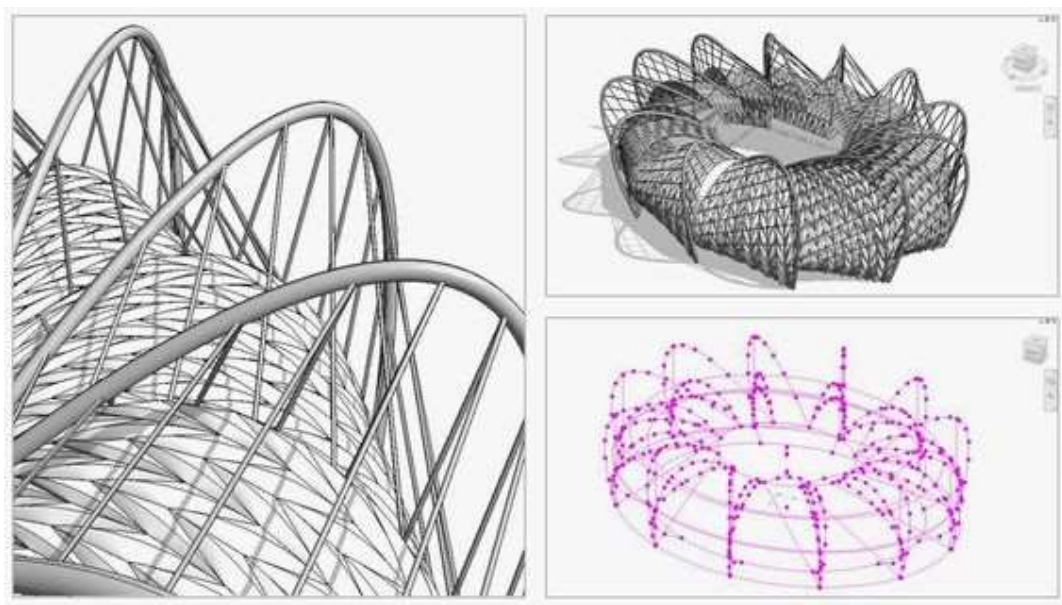

Fig. 6 - Modelado paramétrico de una etapa a partir de la forma de los pétalos de la flor 
Describir en términos matemáticos los fenómenos naturales relacionados con la dinámica no lineal, lleva a los arquitectos a explorar configuraciones más fluidas, vibrantes e inestables, lo que les lleva a utilizar programas cada vez más avanzados de procesamiento, cálculo y simulación, sin los cuales no podrían realizar proyectos tan complejos. Una de las pioneras de esta investigación es Zaha Hadid, que desde el inicio de su carrera ha concebido imágenes complejas y fluidas de espacios y volúmenes, experimentaciones implementadas y apoyadas a lo largo de los años también por el uso de herramientas avanzadas de modelización computacional. Como se verá a continuación.

\section{La modelización tridimensional para el diseño de formas complejas AFRAGOLA STATION NAPOLI, ITALY}

La evolución de las formas siempre ha acompañado el camino de la arquitectura de Zaha Hadid. En una primera época los proyectos se basaban en el imaginario modernista, es decir, en el elemento o elementos planos cuya complejidad venía dada por la intersección de diferentes planos de forma no ortogonal o por bandas siempre formadas por planos regulares e intersecciones circulares, por lo que era posible representar la arquitectura con un modelo físico. La evolución de la complejidad del formulario ha imposibilitado la utilización de modelos en papel como herramienta de estudio y diseño y se ha pasado a la utilización de herramientas digitales. La transición entre el uso del modelo físico para la creación de la arquitectura y la simulación virtual de formas tuvo lugar a principios de los años 2000. El proyecto de la Estación de Alta Velocidad de Afragola es un proyecto de transición entre el MAXXI de Roma y las formas más experimentales de los años siguientes. Situada a unos 12 kilómetros al norte de Nápoles, la estación de Afragola fue diseñada por Zaha Hadid Architects en 2003 para dar cabida a unos 33.000 pasajeros diarios y es la principal estación de intercambio del sur de Italia. La forma de la estación está definida por los recorridos de viajeros, minimizando las distancias entre las grandes entradas y los andenes del tren y conectando todos los espacios con una pasarela que se convierte en el paseo principal: una pasarela pública que conecta las dos partes de la ciudad y alrededor de la cual se ubican todos los servicios. La estructura de hormigón armado, que se extiende a lo largo del trazado curvo de $450 \mathrm{~m}$, sobre el que se fija la estructura de acero que soporta los grandes ventanales de la cubierta, está revestida de corian. La cubierta también incorpora un sistema de paneles solares que, combinado con un cuidadoso estudio de la luz y la ventilación natural, así como sistemas geotérmicos de calefacción/refrigeración, ayudan a minimizar el consumo de energía.

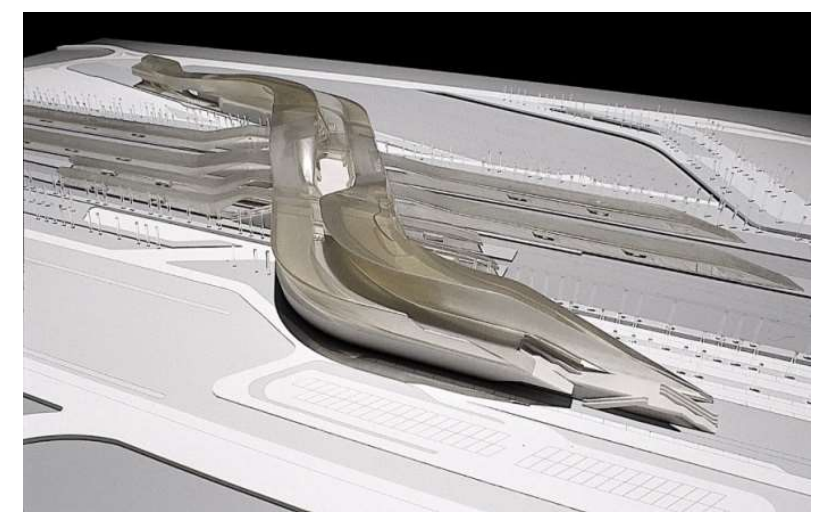

Fig. 7 - Vista de uno de los modelos del concepto del proyecto

El diseño de la estación de Afragola se realizó desde el principio con herramientas diferentes a las utilizadas en trabajos anteriores. Desde hace algún tiempo, la arquitectura del estudio de Hadid fue modelada tridimensionalmente, pero este es uno de los primeros proyectos en los que se utiliza un software de modelado paramétrico de Rhino, un modelador más preciso que los utilizados anteriormente y bastante nuevo en el mercado internacional de la época. Un estudio preliminar de las formas, para la definición de masas y volúmenes, fue realizado con Polygon Modeling por 3ds Max mientras que la definición arquitectónica y estructural del proyecto fue modelada con Rhino. Se han modelado todos las estructuras y ductos principales de la planta, así como las dimensiones de los 
elementos más importantes, para poder comprobar su interferencia con las otras partes del proyecto (detección de colisiones). Los modelos fueron realizados por Zaha Hadid Architects y todos los consultores y especialistas, aunque en su mayor parte fue el equipo de Hadid el que prácticamente reconstruyó las distintas partes y verificó la coordinación del conjunto. En este sentido, no podemos hablar realmente de BIM porque, en 2003, tanto el concepto de BIM como, sobre todo, la instrumentación disponible, no permitieron fluidificar el proceso de transición de la información digital del proyecto. [15]

\section{El BIM para el diseño de formas complejas y para la optimización de los componentes PHOENIX INTERNATIONAL MEDIA CENTER BEIJING, CHINA}

El Phoenix International Media Center es una arquitectura muy particular, hecha a partir de una envoltura de vidrio y acero que proviene de un volumen creado siguiendo la forma geométrica de la cinta de Möbius. Con una superficie bruta de 65.000 metros cuadrados y una altura de $55 \mathrm{~m}$, consigue contener todo el espacio necesario para la nueva sede de la emisora china Phoenix Televisión, a pesar de que el uso previsto conlleva el reto de combinar funciones muy diferentes: grandes espacios para estudios de dirección y emisión de televisión y edificios de varias plantas para oficinas. BIAD, el estudio de diseño, resolvió este problema insertando las áreas de transmisión en las partes más anchas de la cinta de Möbius y el edificio de oficinas de varios pisos en la zona más estrecha y alta. El armazón está formado por una estructura principal de acero y una piel de vidrio y acero, que encierra los dos edificios, conectados por pasarelas de acero blanco en varias plantas. El proyecto fue premiado en la conferencia de la Autodesk University 2016, en los Premios AEC a la Excelencia en la sección "Edificios", en reconocimiento a los proyectos que "demuestran un uso ejemplar del Modelado de Información de Edificios" y que "incluyen categorías de edificios que abrazan el futuro de la creación de AEC (sector de la construcción) a través del uso de tecnologías BIM innovadoras y complejas".

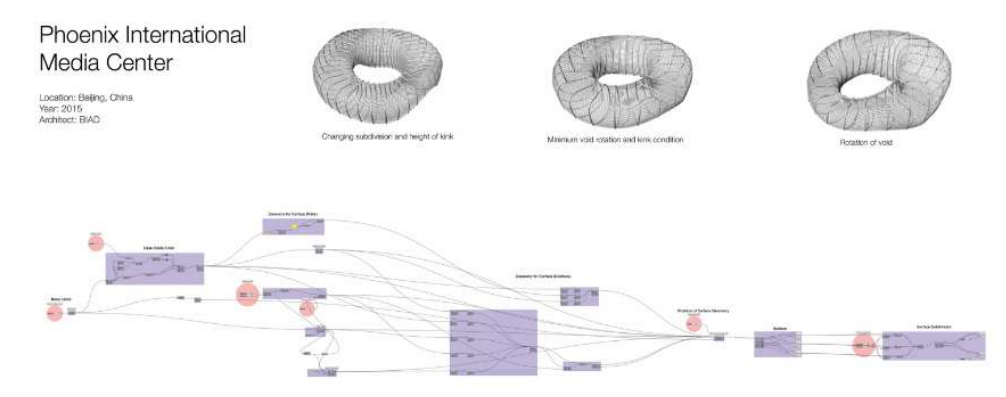

Fig. 8 - Diagrama paramétrico del modelo para el ajuste de la forma

La complejidad del Phoenix International Media Center ha creado una serie de retos interesantes, tales como la gestión del impacto con los edificios vecinos existentes, o cómo diseñar un edificio energéticamente eficiente. El BIM ayudó a gestionar sistemáticamente todos estos aspectos: el modelo permitió, por ejemplo, simular sombras y reflejos no deseados en edificios cercanos, ayudando a encontrar la posición más adecuada para la parte superior del edificio. Partiendo del modelo arquitectónico BIM se ha podido diseñar la estructura metálica, insertando en la base de datos BIM también todos los datos necesarios para la realización de los componentes en fábrica y para el montaje en obra; la modelización de la forma y las curvaturas de todos los elementos metálicos también ha permitido diseñar todos los puntos de unión de las diferentes piezas. 


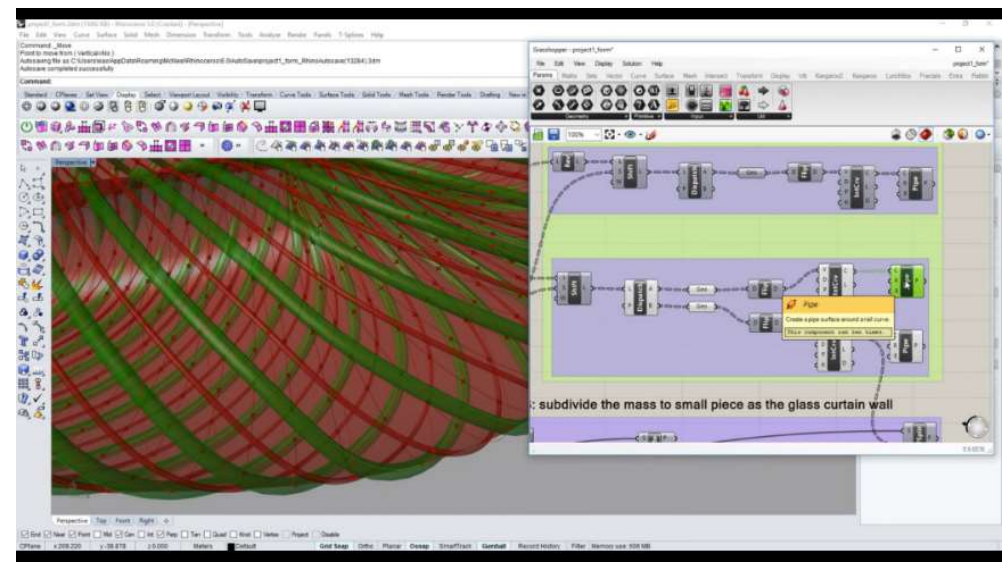

Fig. 9 - Modelado paramétrico de la estructura primaria y secundaria

La fachada presenta dos niveles estructurales fácilmente identificables: uno que crea secciones verticales de la banda, caracterizadas por perfiles más grandes y que constituye la estructura primaria de la envolvente, mientras que el otro sigue el curso del anillo con un escaneado más delgado, es la estructura secundaria. Gracias al modelado paramétrico también ha sido posible realizar comprobaciones y optimizaciones en las curvaturas de los paneles tratando de limitar al máximo los costes y obteniendo un número de 5.180 paneles diferentes. Según los cálculos del BIAD, la elección de utilizar BIM ha permitido alcanzar importantes objetivos, tanto de ahorro económico como de conocimiento en el campo de la modelización informativa. Se ha calculado un ahorro de más de 870.000 dólares, debido en parte a la optimización del material y en parte a los costes derivados de la reducción de los tiempos dedicados al diseño e instalación del muro cortina. Además, se ha calculado un ahorro adicional de más de 7.400 .000 dólares por el uso de placas de vidrio plano en lugar de vidrio curvado en la construcción del muro cortina, resultado de la optimización del encofrado en el estudio de las inclinaciones de las caras de la superficie de la envolvente. [16]

\section{BIBLIOGRAFÍA}

[1] M. Gell-Mann, II quark e il giaguaro. Avventura nel semplice e nel complesso, Bollati-Boringhieri, 1996.

[2] E. Morin, «Le vie della complessità,» in La sfida della complessità, Milano, Feltrinelli, 1985.

[3] S. Rossella, II processo biomimetico sistemico nel progetto tecnologico di architettura, Napoli: Dottorato di Ricerca in Tecnologia dell'Architettura e Rilievo e Rappresentazione dell'Architettura e dell'Ambiente ciclo XXVII, 2015.

[4] M. Pawlyn, «TED - Ideas worth spreading,» TED, $2010 . \quad$ [Online]. Available: https://www.ted.com/talks/michael_pawlyn_using_nature_s_genius_in_architecture?language=en.

[5] M. A. Curran, Life Cycle Assessment: An International Experience., Wiley Online Library, 2004.

[6] W. McDonough e M. Braungart, Dalla culla alla culla. Come conciliare tutela dell'ambiente, equità sociale e sviluppo, Torino: Blu Edizioni, 2003.

[7] C. Eastman, BIM Handbook: A Guide to Building Information Modeling for Owners, Managers, Designers, Engineers and Contractors, Wiley, 2011.

[8] V. Villa, «ll Building Information Modeling,» in Manule dell'Ingegnere Civile, Milano, HOEPLI, 2017.

[9] M. Coppola, La maniera biomimetica, Roma: d editore, 2016.

[10] F. Capra e L. P. L., Vita e natura. Una visione sistemica, San Sepolcro: Aboca, 2014.

[11] Puig-Boada, El Pensament de Gaudì (Compilacaò de textos i comentaris), Barcelona: Collegi d'Arquitectes de Catalunya, 1981.

[12] P. Portoghesi, Natura e architettura, Milano: Skira, 1999.

[13] M. Sgambelluri, «Practically Dynamo: Practical Uses for Dynamo within Revit,» Autodesk University, 2014.

[14] K. Taki, A conversation with Toyo Ito.

[15] G. M. Di Giuda e V. Villa, «Afragola Station Napoli, Italy. La modellazione tridimensionale per la progettazione di forme complesse,» Arketipo, n. 113, pp. 132-135, 2017.

[16] G. M. Di Giuda e V. Villa, «ll BIM per la progettazione di forme complesse e per l'ottimizzazione dei componenti,» Arketipo, n. 121, pp. 142-145, 2018.

[17] A. Saggio, Introduzione alla Rivoluzione Informatica in Architettura, Roma: Carocci Editore, 2007.

[18] L. Galofaro, Eisenman digitale. Uno studio dell'era elettronica, Torino: Testo\&Immagine, 1999.

[19] T. Ito, to 200 Architettura come processo, Milano: Electa, 2005.

[20] P. Mello, Ito Digitale. Nuovi media, Nuovo reale, Roma: Edilstampa, 2008.

[21] C. Dresser, The art of decorative design, 2001. 